\title{
Attention Deficit Hyperactivity Disorder (Adhd) Detection Methods
}

\author{
M. SHERIFF, R. GAYATHRI
}

\begin{abstract}
Attention Deficit Hyperactivity Disorder (ADHD) is one of the most common mental-health disorders, affecting around 5\%-10\% of school-age children. This paper details about various methodologies for detecting and diagnosing the ADHD disease in patients using different soft computing and deep learning techniques. The limitations of advantages of each ADHD method were discussed in detail with its corresponding simulation results. The feature extraction method and its training with classification procedure for each conventional ADHD method were illustrated in detail.
\end{abstract}

Keywords: ADHD, Disorder, Features, Classifications, Diagnosing.

\section{INTRODUCTION}

Miguel Ángel Bautista et al. (2016) used gesture recognition methodology for detecting and identifying the natural behavioral functionalities of ADHD persons using the aid of embedded system. The authors applied dynamic time warping algorithm for the detection of gestures for specific patterns for ADHD recognition. Gaussian mixture models were constructed for each class of behavioral activities of ADHD persons for differentiating the normal functionalities with abnormal functions. The authors extracted Head Turning Behavioral Pattern Features from different set of functional behavior of the persons and they were classified using dynamic time warping algorithm. The performance of this proposed methodology was analyzed with respect to random mean and error function for different set of classes. Fig. 1 shows the ADHD recognition using gesture system.

Liang zou et al. (2017) detected and identified ADHD activities of the patients using deep learning algorithms such as Convolution Neural Networks (CNN). The features were extracted from the MRI image and then they are classified with SOFTMAX classier. The max pooling algorithm was used in CNN classification architecture for maximizing the classification rate of the ADHD disease detection system. The authors achieved $69.15 \%$ of average classification accuracy for ADHD detection in patients using this $\mathrm{CNN}$ classification algorithm. The authors tested their propose ADHD detection algorithm on 239 persons MRI dataset and achieved this classification accuracy. Fig. 2 shows the proposed ADHD detection system using CNN and SOFTMAX classification approach.

Revised Manuscript Received on July 22, 2019.

M.Sheriff, Assistant Professor, Dept. of Electronics and Communication Engineering, VelTech,Chennai. Email:sheriff.emsys@gmail.com R.Gayathri, Associate Professor\& Research Supervisor, Dept. of Electronics and Communication Engineering, Sri Venkateswara College of Engineering, Chennai. Email: rgayathri@svce.ac.in

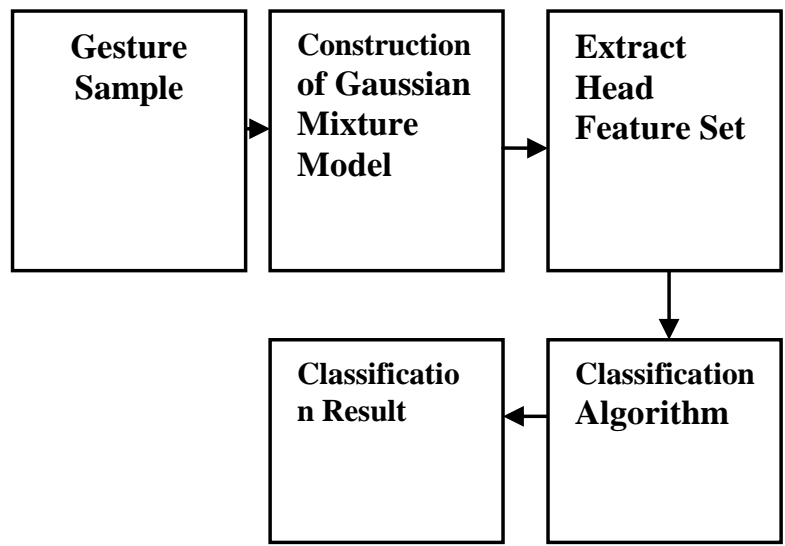

Figure: 1 ADHD Recognition Using Gesture System
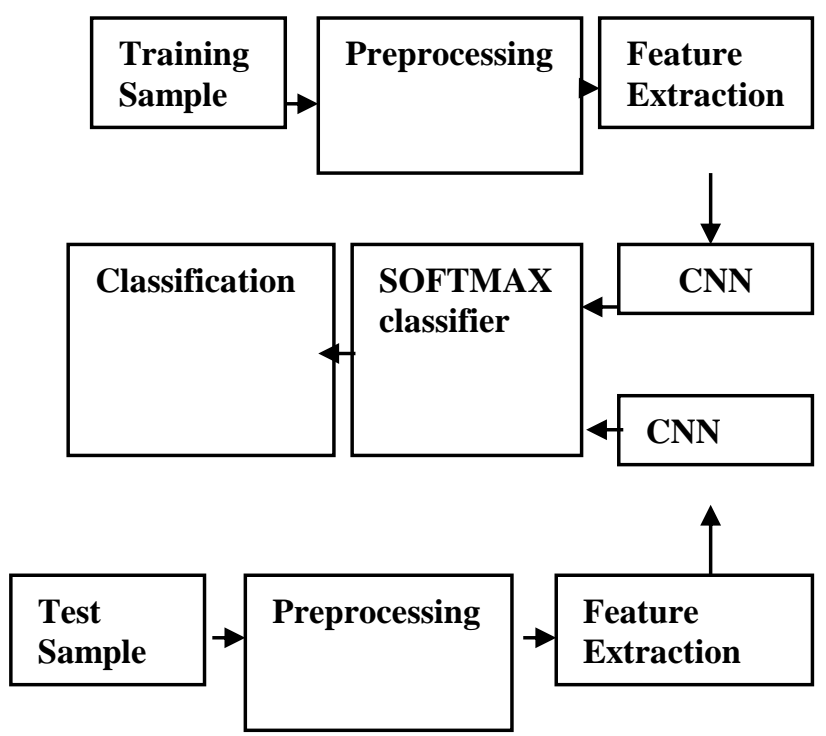

Figure: 2 ADHD Detection System Using CNN \& SOFTMAX Classification Approach

\section{SURVEY ON DECOMPOSITION AND CLASSIFICATION METHODS FOR ADHD DETECTION}

Sudha et al. (2017) used Modified Dual Tree Complex Wavelet Transform (MDTCWT) method for identifying the gait pattern of ADHD children's. The authors used background subtraction algorithm for subtracting the foreground object from background object for identifying the behavior of the children's. Then, the variations in pixel by pixel Sciences Publication

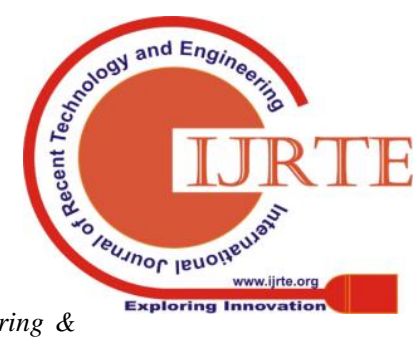


with respect to its surrounding pixel; variations were analyzed for detecting the gait of different functional activities of the ADHD patients. The authors achieved $82 \%$ of classification rate for their proposed gait classification methodology for ADHD patients. Fig. 3 shows the Gait classification using Modified Dual Tree Complex Wavelet Transform (MDTCWT) method.

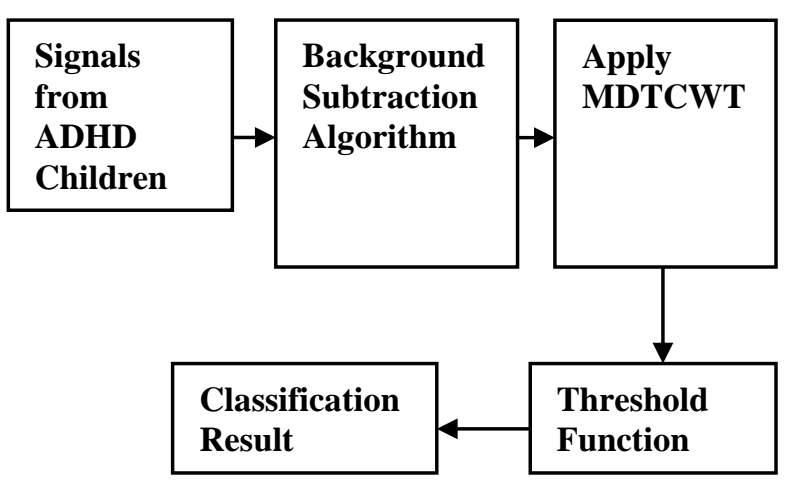

Figure: 3 Gait Classification Using Modified Dual Tree Complex Wavelet Transform (MDTCWT) Method

Alaa Eddin Alchalabi et al. (2018) used Radial Basis Function Support Vector Machine (SVM) classification algorithm for classifying the functional activities of ADHD persons. The authors obtained $98.62 \%$ of average classification accuracy on their proposed methodology on EEG signals of the different patients. This classification accuracy was obtained by testing their proposed method on EEG signals when the game was applied on the patients.

Fivefold cross validation method was applied on the classification results to validate the classification results of the proposed ADHD detection method. The authors obtained average precision of $64 \%$ and an average recall rate of $60 \%$ for different set of sample tests. Fig.4 shows the ADHD detection using Radial Basis Function Support Vector Machine (SVM) classification approach.

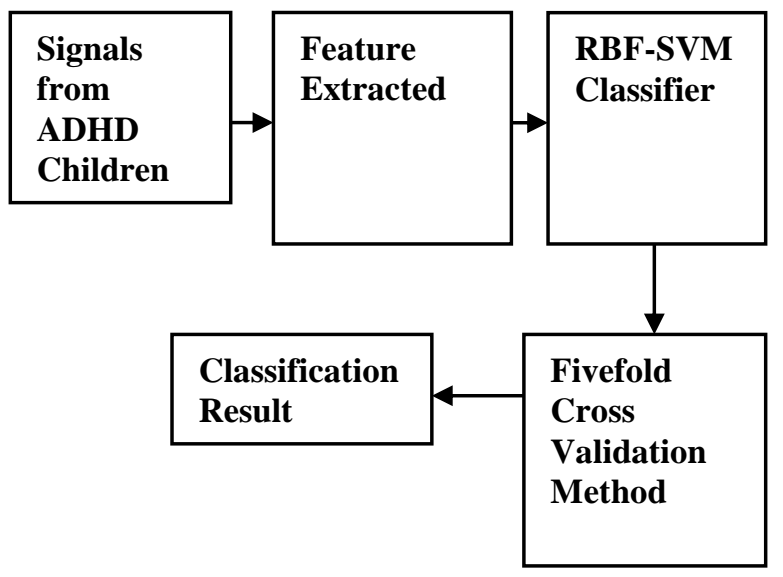

Figure: 4 ADHD detection using Radial Basis Function Support Vector Machine (SVM) Classification Approach

\section{SURVEY ON FEATURE EXTRACTION METHODS FOR ADHD DETECTION}

Gulay CICEK et al. (2018) used Local and Global Features for differentiating the functional behavior and activities of the
ADHD persons with normal persons. In this work, MRI images from different 26 persons under different criteria were collected and the proposed method was applied on these collected MRI images to validate the effectiveness of the proposed ADHD method. The author's derived shape and texture features from the different regions from MRI images and these features were classified using k-nearest neighborhood algorithm (KNN) and naive Bayes classification approaches.

The authors additionally included Grey Level Co-occurrence matrix (GLCM) feature set with the existing global feature set in order to maximize the classification rate of the proposed ADHD detection system. Finally, fivefold cross validation method was applied on the results to validate the effectiveness of the proposed method. The proposed method stated in this paper reaches $100 \%$ of average classification accuracy when the extracted features from the MRI image can be integrated into single feature vector. Fig. 5 shows the ADHD detection method using k-nearest neighbor's algorithm (KNN) and naive Bayes classification approach.

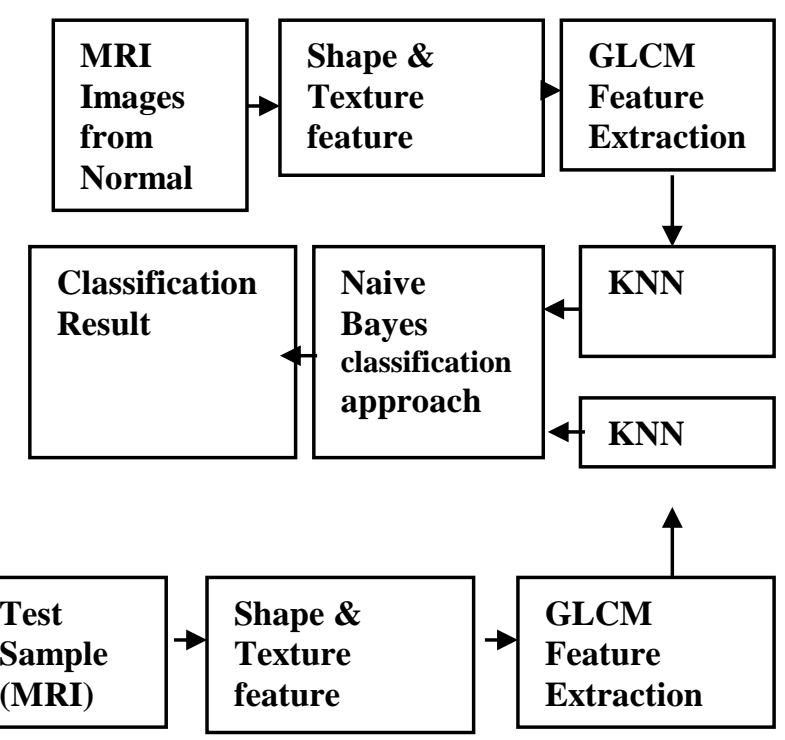

Figure: 5 ADHD detection Method Using K-Nearest Neighbor's Algorithm (KNN) and Naive Bayes Classification Approach.

Eloyan et al. (2012) used MRI imaging techniques to detect and diagnose the ADHD disorders in the persons. The authors obtained $68.5 \%$ bof average classification accuracy on the real time data sets. Fivefold cross validation method was applied on the test MRI images in order to validate the achieved results.

Matthew et al. (2012) also used MRI imaging methods for identifying the functional activities of the ADHD patients with respect to the normal persons. The authors obtained $72.1 \%$ bof average classification accuracy on the real time data sets. Fivefold cross validation method was applied on the test MRI images in order to validate the achieved results.

Table.1 shows the comparisons of ADHD detection methods with respect to classification accuracy. From Table 1, it is 
clear that the method Sudha et al. (2017) using Modified Dual Tree Complex Wavelet Transform (MDTCWT) obtained $82 \%$ of classification accuracy and Gulay CICEK et al. (2018) using Radial Basis Function Support Vector Machine (SVM) classification approach obtained $100 \%$ of classification accuracy for ADHD detections.

Table:1 Comparisons of ADHD Detection Methods with Respect to Classification Accuracy

\begin{tabular}{|l|l|l|}
\hline Authors & Methodologies & $\begin{array}{l}\text { Classificatio } \\
\mathrm{n} \text { accuracy } \\
(\%)\end{array}$ \\
\hline $\begin{array}{l}\text { Miguel } \\
\text { Angel } \\
\text { Bautista et } \\
\text { al. (2016) }\end{array}$ & $\begin{array}{l}\text { Head Turning Behavioral } \\
\text { Pattern Features }\end{array}$ & 61.7 \\
\hline $\begin{array}{l}\text { Liang zou et } \\
\text { al. (2017) }\end{array}$ & $\begin{array}{l}\text { Convolutional Neural } \\
\text { Networks (CNN) }\end{array}$ & 69.15 \\
\hline $\begin{array}{l}\text { Sudha et al. } \\
\text { (2017) }\end{array}$ & $\begin{array}{l}\text { Modified Dual Tree } \\
\text { Complex Wavelet } \\
\text { Transform (MDTCWT) }\end{array}$ & 82 \\
\hline $\begin{array}{l}\text { Alaa Eddin } \\
\text { Alchalabi et } \\
\text { al. (2018) }\end{array}$ & $\begin{array}{l}\text { Modified Dual Tree } \\
\text { Complex (ransform (MDTCWT) } \\
\text { method }\end{array}$ & 60 \\
\hline $\begin{array}{l}\text { Gulay } \\
\text { CICEK et al. } \\
\text { (2018) }\end{array}$ & $\begin{array}{l}\text { Radial Basis Function } \\
\text { Sum) classification } \\
\text { approach }\end{array}$ & 100 \\
\hline $\begin{array}{l}\text { Eloyan et al. } \\
\text { (2012) }\end{array}$ & $\begin{array}{l}\text { MRI Imaging method } \\
\text { al. (2012) }\end{array}$ & 68.5 \\
\hline
\end{tabular}

Fig. 6 illustrates the graphical comparisons of ADHD detection methods with respect to classification accuracy.

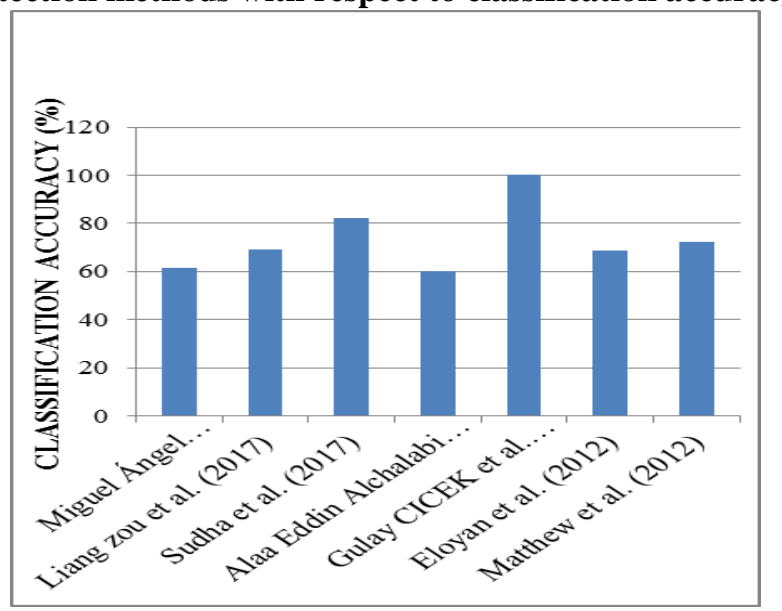

Figure: 6 Graphical Comparisons of ADHD Detection Methods

\section{CONCLUSIONS}

This paper details about various methodologies for detecting and diagnosing the ADHD disease in patients using different soft computing and deep learning techniques. The limitations of advantages of each ADHD method were discussed in detail with its corresponding simulation results. Sudha et al. (2017) using Modified Dual Tree Complex Wavelet Transform (MDTCWT) obtained $82 \%$ of classification accuracy and Gulay CICEK et al. (2018) using Radial Basis Function Support Vector Machine (SVM) classification approach obtained $100 \%$ of classification accuracy for ADHD detections.

\section{REFERENCES}

1. Miguel Ángel Bautista, Antonio Hernández-Vela, Sergio Escalera, “A Gesture Recognition System for Detecting Behavioral Patterns of ADHD", IEEE Transactions on Cybernetics, Vol. 46, no. 1, 2016,pp.136-148.

2. Liang zou, Jiannan zheng, Chunyan miao, Martin J. mckeown, and Z. Jane wang, "3D CNN Based Automatic Diagnosis of Attention Deficit Hyperactivity Disorder Using Functional and Structural MRI”, IEEE Access, 2017

3. Sudha D, M. Pushpa Rani, "Gait Classification for ADHD Children Using Modified Dual Tree Complex Wavelet Transform”, Gait Classification for ADHD Children Using Modified Dual Tree Complex Wavelet Transform, 2017.

4. Alaa Eddin Alchalabi , Shervin Shirmohammadi, "FOCUS: Detecting ADHD Patients by an EEG-Based Serious Game", IEEE Transactions on Instrumentation and Measurement, Vol. 67, no. 7, 2018, pp.1512-1520.

5. Gulay CICEK, Aydin AKAN, Baris METIN, "Detection of Attention Deficit Hyperactivity Disorder Using Local and Global Features", PLOS ONE, 2018.

6. Eloyan, A., Muschelli J., Nebel, H, Han, F., Zhao T., Barber, A., Joel S., James J., Mostofsky, S., \& Brian Caffo “Automated diagnoses of attention deficit hyperactive disorder using magnetic resonance imaging", Frontiers in Systems Neuroscience, vol.6, No.61, 2012.

7. Matthew, R., Sidhu, G., Greiner, R., Asgarian, N., Bastani, M., Silverstone, P., Greenshaw, A., \& Serdar M., "ADHD-200 Global Competition: diagnosing ADHD using personal characteristic data can outperform resting state fMRI measurements", Frontiers in Systems Neuroscience, vol.6, no.69, 2012.

8. H.-I. Suk, S.-W. Lee, D. Shen, and The Alzheimer's Disease Neuroimaging Initiative, "Hierarchical feature representation and multimodal fusion with deep learning for AD/MCI diagnosis," NeuroImage, vol. 101, pp. 569582, Nov. 2014.

9. C.-W. Chang, C.-C. Ho, and J.-H. Chen, “ADHD classification by a texture analysis of anatomical brain MRI data," Frontiers Syst. Neuroscience., vol. 6, p. 66, Sep. 2012.

10. Q.-H. Zou et al., "An improved approach to detection of amplitude of low-frequency fluctuation (ALFF) for resting-state fMRI: Fractional ALFF," J. Neuroscience. Methods, vol. 172, no. 1, pp. 137_141, 2008.

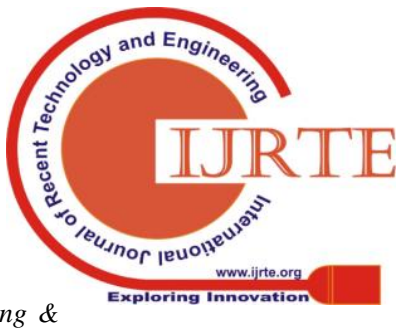

